\title{
Role of Rat Intestinal Glucoamylase in Glucose Polymer Hydrolysis and Absorption
}

\author{
MOHAMMAD A. K. AZAD AND EMANUEL LEBENTHAL \\ International Institute for Infant Nutrition and Gastrointestinal Disease, Children's Hospital of Buffalo, New \\ York 14222, and Department of Pediatrics, Hahnemann University, Philadelphia, Pennsylvania 19102-1192
}

\begin{abstract}
Rice starch is a main source of energy in many lesser developed countries. We studied different chain-lengths of rice glucose polymers (GP) to evaluate their possible use in feeding infants in developing countries. The initial $\mathrm{GP}$ of rice $(\mathrm{G} 1=4.6, \mathrm{G} 2=4.5, \mathrm{G} 3=15.4, \mathrm{G} 4$ $=7.3, \mathrm{G} 5=17.4, \mathrm{G} 6-\mathrm{G} 9=9.61$ and $>\mathrm{G} 9=31.3 \%$ ) was analyzed by HPLC and then separated in a Bio-Gel P-2 column and compared to its short-chain GP of rice $(\mathrm{G} 2=$ $22.7, \mathrm{G} 3=28.2, \mathrm{G} 4=14.0, \mathrm{G} 5=16.6, \mathrm{G} 6=11.6, \mathrm{G} 7-$ G9 $=6.9 \%)$, long-chain $\mathrm{GP}$ of rice $(>\mathrm{G} 9=100 \%)$, and Dglucose. Intraduodenal bolus infusion of $10 \%$ solution of short-chain rice GP when compared with long-chain rice GP, the initial rice GP, or D-glucose showed significantly higher values at peak absorption time (0 to $30 \mathrm{~min}$ ) in the portal venous blood glucose response. The portal venous glycemic response of short-chain rice GP compared with D-glucose was as follows: $2.5 \pm 0.1$ versus $2.0 \pm 0.2 \mathrm{~cm}^{2}$, area under the portal blood glucose curve at $0-30 \mathrm{~min}(p$ $<0.01$ ). Glucoamylase, the key enzyme for brush-border hydrolysis of short-chain GP, was assessed with a newly modified glucoamylase assay using GP G5-G8 as substrate. Our finding of faster glucose absorption with shortchain rice GP compared with isocaloric D-glucose might have important physiologic implications for carbohydrate absorption. The osmolality of short-chain rice GP is nearly one-fourth that of glucose. This might have important bearing in the design of infant feeding where increased caloric density with low osmolality is desirable. (Pediatr Res 28: 166-170, 1990)
\end{abstract}

\section{Abbreviations}

GP, glucose polymer

Rice starch is one of the main sources of energy in food available in many lesser developed countries. Starch (amylose and amylopectin) is rapidly hydrolyzed by salivary and pancreatic amylases to short GP, maltotriose, maltose, and glucose (1). The brush-border glucoamylase, maltase, and isomaltase act on the products of luminal starch digestion releasing glucose (2).

During the first 4 to 6 mo of life, the key enzyme for starch digestion, pancreatic amylase, is very scarce or nonexistent (3, 4). In addition, brush-border disaccharidases, because of their predominant localization in the proximal small intestine where the mucosal injury from protracted diarrhea of infancy can be

Received August 29, 1989; accepted March 27, 1990

Correspondence: Emanuel Lebenthal, M.D. The International Institute for Infant Nutrition \& Gastrointestinal Disease, Department of Pediatrics, Hahnemann University, Broad and Vine, Philadelphia, PA 19102-1192.

Study was supported by a cooperative agreement with USAID. DPE No. 5940A-004019-00. M.A.K.A. was temporarily assigned to IIINGD from ICDDR.B. Dhaka, and was supported by a grant from USAID the most severe (5), are likely to be diminished during chronic diarrhea in infancy (6-8).

It has been suggested that the availability of glucoamylase in the small intestine can play a role in GP digestion and absorption in early life. Moreover, intestinal glucoamylase is distributed throughout the small intestinal mucosa and tends to be more resistant to intestinal injury than the disaccharidases (9). However, it is not clear whether rice starch hydrolyzed to shorter polymers of glucose will be better absorbed in the small intestinal mucosa in the first $6 \mathrm{mo}$ of life, especially in those with prolonged mucosal injury in persistent diarrhea of infancy.

The aim of our study was to evaluate the effect of different chain-lengths of GP generated from rice on the glucose response in the portal vein of a rat model. Perfusion studies were performed with the initial GP of rice, short-chain GP of rice, longchain GP of rice, and D-glucose. The GP were separated and prepared from the initial GP of rice using a modified system of gel permeation chromatography and determined by HPLC.

The role of glucoamylase in the specific hydrolysis and absorption of the GP was examined. The study used a new substrate of a purified short-chain GP (chain-length G5-G8) in addition to glucogen for the glucoamylase assay.

\section{MATERIALS AND METHODS}

Animals. Adult Sprague-Dawley rats, weighing 250-350 g, were used for all experiments.

$G P$. GP are a heterogenous group of linear chains of glucose residues, linked predominantly by $\alpha-1,4$ glucosidic bonds. The nomenclature used in this report is as follows: G1, free glucose; G2, maltose; G3, maltotriose; G4, maltotetraose; G5, maltopentose; G6, maltohexose; G7. maltoseptose; G8, maltoctaose; G9. maltonanose; $>\mathrm{G} 9$, GP of longer chain-length than 9 glucose units. The initial GP preparation from rice (donated by Mead Johnson, Evansville, IN) was analyzed by HPLC and composed of $\mathrm{G} 1=4.6, \mathrm{G} 2=14.5, \mathrm{G} 3=15.4, \mathrm{G} 4=7.3$, G5 = 17.4, G6$\mathrm{G} 9=9.61$, and $>\mathrm{G} 9=31.3 \%$ (Fig. $1 A$ ). Samples for HPLC analysis were passed through three thicknesses of millipore filters. Twenty $\mu \mathrm{L}$ of the filtrate were then applied to an Aminex HPX42A HPLC column (Bio-Rad Laboratories, Richmond, CA) for analysis of initial GP of rice, short-chain GP, long-chain GP, corn GP, and glucoamylase substrate at $85^{\circ} \mathrm{C}$ using specially purified water (HPLC grade, Fisher Scientific, Fairlawn, NJ) as the mobile phase. The profile depicting the individual products was analyzed with a computer-aided data master system from Gilson Medical Electronics (Middleton, WI). Individual components were quantitated as areas under the elution peaks and expressed as percent of total GP measured. GP profiles were integrated using a computer program. Individual components that were integrated include G1, G2, G3, G4, G5, G6, G7, G8, G9, and $>$ G9. The relative percent of each peak was calculated as a fraction of the sum totals.

Short-chain GP and long-chain GP were separated and prepared from the initial rice GP using a modified system of gel 
Initial Rice GP
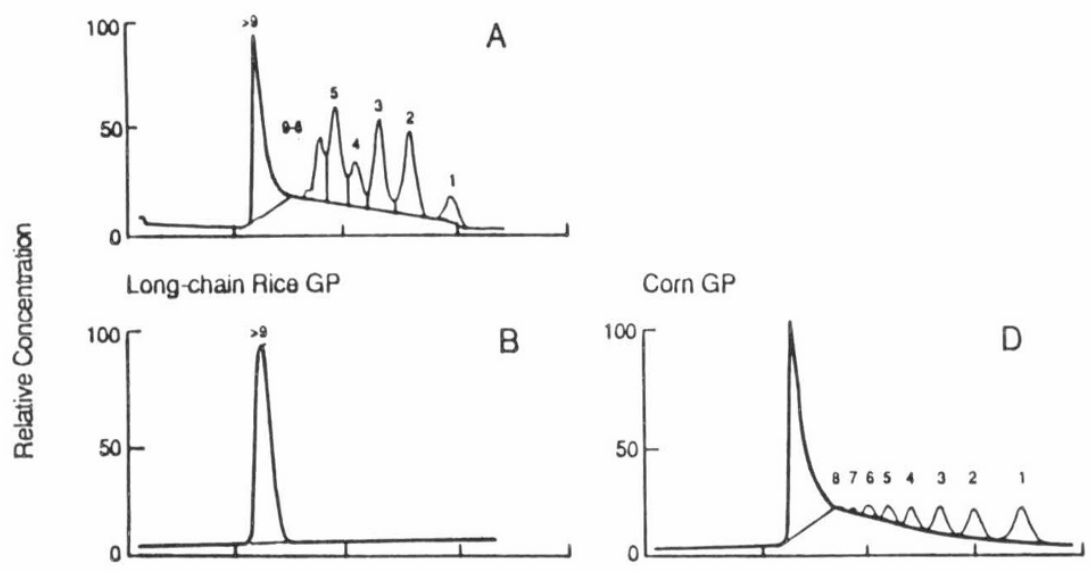

Short-chain Rice GP

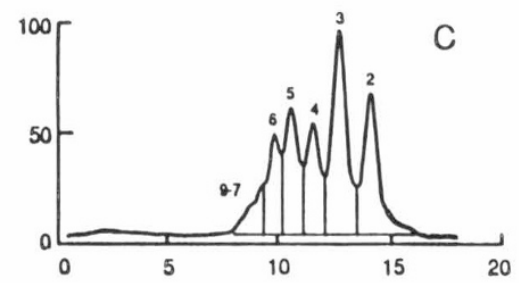

Glucoamylase Substrate

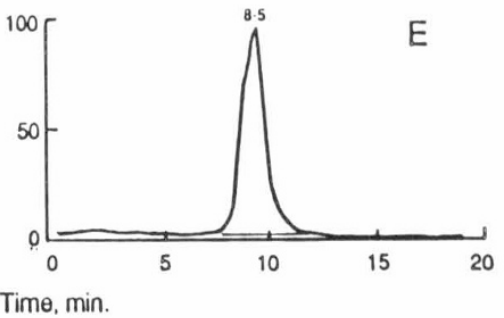

Fig. 1. Representative chromatograms of the initial rice GP $(A)$, long-chain rice GP $(B)$, short-chain rice GP $(C)$, corn GP $(D)$, and glucoamylase substrate (G5-G8) (E).

permeation chromatography (10). This system separated and classified components on the basis of molecular size. A waterjacketed glass column $(2.5 \mathrm{~cm} \times 90 \mathrm{~cm})$ was packed with a preswollen aqueous slurry of Bio-Gel P-2, 200-400 mesh (BioRad Laboratories). When almost fully packed, a rubber stopper (attached to a closed hydrostatic pressure system for elution water delivery) was fitted to take up any dead volume at the top of the column. The jacket temperature was slowly raised to $65^{\circ} \mathrm{C}$ while the column was eluted with deaerated water. Once the column was fully equilibrated (after $\sim 24 \mathrm{~h}$ ), the elution with water was continued and 3 -mL aliquots of suitably diluted samples were introduced into the elution stream.

The molecular sizes of unknown components of the sample were identified from spectrophotometric reading at $190 \mathrm{~nm}$ wavelength and their elution position was compared with a chart for standards.

The eluate fractions that contained G2-G9 GP and > G9 GP were concentrated, lyophilized, and validated by HPLC (Rainin, Woburn, MA). The same procedure was performed to separate and purify the corn GP of G5 to G8 that served as a substrate for determination of glucoamylase activity.

Osmolality measurements of test GP solutions including Dglucose were done by vapor pressure osmometer (Model 5100B, Wescor, Inc. Logan, UT). Osmolality of $10 \%$ solutions of Dglucose, the initial rice-GP, short-chain GP, and long-chain GP were $561,158,161$, and $212 \mathrm{mosmol} / \mathrm{kg}$, respectively.

Infusion technique. After an overnight fast, the animals were anesthetized by intraperitoneal injection of pentobarbital sodium (70 mg/ $\mathrm{kg}$ body wt) and maintained with a quarter of the initial dosage. The abdomen was opened by a midline incision and the GP was infused through a 21 -gauge needle intraduodenally $4 \mathrm{~cm}$ proximal to the ligament of Treitz as a $10 \%$ solution bolus, at a dose of $0.5 \mathrm{gm} / \mathrm{kg}$ body wt within $1 \mathrm{~min}$. Biliary and pancreatic secretions were not excluded from the infused segment. The intestines were kept moist with warm saline-soaked gauze, and the animals were kept warm with an overhead infrared lamp.

Determination of portal venous blood glucose levels. Fifty to $100 \mu \mathrm{L}$ of portal venous blood were drawn at 30 -min intervals from 0 to $120 \mathrm{~min}$ in each experiment. Blood glucose was estimated by the glucose oxidase method by Glucometer, Ames, IA. The machine was equilibrated with low and high calibrators before the start of the project and when batteries were changed. Area under the portal venous blood glucose curve was defined as the postinfusional rise above baseline over time.

Preparation of mucosal homogenate. After killing the rats at the end of the study, a $15-\mathrm{cm}$ jejunal segment distal to the ligament of Treitz was taken out, rinsed with cold saline 6 times to get rid of contaminating pancreatic $\alpha$-amylase as much as possible, and then opened by longitudinal incision. The mucosa was scraped off gently using a glass slide and stored at $-70^{\circ} \mathrm{C}$. Before enzymatic determinations, the jejunal scrapings were homogenized by hand using a glass tissue homogenizer with 100 volumes of distilled water at $4^{\circ} \mathrm{C}$.

Enzymatic determinations. Glucoamylase activity was determined by a modification of the method used by Eggermont $e t$ al. (11). The substrate solutions contained $0.3 \mathrm{~g}$ of GP (G5-G8) or glycogen plus $0.025 \mathrm{gm} \mathrm{BSA}$ in $10 \mathrm{~mL}$ of $0.15 \mathrm{M}$ sodium citrate buffer at $\mathrm{pH} 5.4$ (to inhibit the pancreatic $\alpha$-amylase). Activities measured were expressed as $\mu \mathrm{g}$ of glucose liberated per assay mixture after the designated time of incubation. Disaccharidase activities were assayed by the method of Townley et al. (12). Glucose was assayed using the diagnostic statzyme reagent (Worthington Biochemical Co., Freehold, NJ). Protein was determined by the technique of Lowry et al. (13).

Statistics. All results are expressed as mean \pm SEM. Differences in mean values between groups were determined by an unpaired $t$ test. $p$ values of less than 0.05 were considered significant.

\section{RESULTS}

$G P$. Figure 1 depicts representative chromatograms of the initial GP from rice (Fig. $1 A$ ), long-chain GP of rice (Fig. $1 B$ ), short-chain GP of rice (Fig. 1C), corn GP (Fig. 1D), and the corn GP G5-G8 serving as glucoamylase substrate. The initial $\mathrm{GP}$ from rice was composed of $\mathrm{G} 1=4.6, \mathrm{G} 2=14.5, \mathrm{G} 3=15.4$, $\mathrm{G} 4=7.3, \mathrm{G} 5=17.4, \mathrm{G} 6-\mathrm{G} 9=9.6$, and $>\mathrm{G} 9=31.3 \%($ Fig 
1A). The long-chain GP of rice was composed of $>\mathrm{G} 9=100 \%$ (Fig. $1 B$ ). The short-chain GP of rice was composed of $\mathrm{G} 2=$ $22.7, \mathrm{G} 3=28.2, \mathrm{G} 4=14.0, \mathrm{G} 5=16.6, \mathrm{G} 6=11.6, \mathrm{G} 7-\mathrm{G} 9=$ $6.9 \%$ (Fig. 1C).

Infusion of GP and portal venous glucose response. All GP and glucose produced maximal portal venous glucose peak at $30 \mathrm{~min}$ (Fig. 2). After the infusion of the test GP and glucose, portal blood glucose peaked from $63.0 \pm 4.8$ to $94.4 \pm 6.5 \mathrm{mg} / \mathrm{dL}$ for initial GP of rice, $60.2 \pm 1.5$ to $108.2 \pm 2.0 \mathrm{mg} / \mathrm{dL}$ for shortchain GP, $63.4 \pm 2.0$ to $88.2 \pm 3.5 \mathrm{mg} / \mathrm{dL}$ for long-chain GP, and $68.0 \pm 2.7$ to $105.6 \pm 1.1 \mathrm{mg} / \mathrm{dL}$ for D-glucose. Portal venous blood glucose then returned to a level that was not significantly different from the baseline at $120 \mathrm{~min}$ for each of the carbohydrates studied.

Glycemic response. The glycemic response measured as the mean of the area under the curve revealed a significantly higher value for short-chain GP of rice compared with the initial GP of rice, long-chain rice GP, and D-glucose during the peak absorption time, namely 0 to $30 \mathrm{~min}$ (Table 1 ). At $120 \mathrm{~min}$, the glycemic response values for both short-chain GP and D-glucose did not show any significant difference.

The infusion of the initial GP of rice and the long-chain GP was associated with a wide SD of the portal venous blood glucose levels, whereas those for short-chain GP and glucose revealed a narrow SD.

Glucoamylase activity determination using G5 to G8 as substrate. The adequacy of glucoamylase (E.C. 3.2.1.3) assay used in our study was checked by several methods. The use of an optimal substrate of GP, G5 to G8 units, (Fig. 1E) to determine the glucoamylase activity has a great advantage over the glycogen that was used in previous studies $(9,11)$. Figure 3 compares the time course of glucoamylase activity using G5 to G8 units as substrate with the time course when using glycogen. The sp act

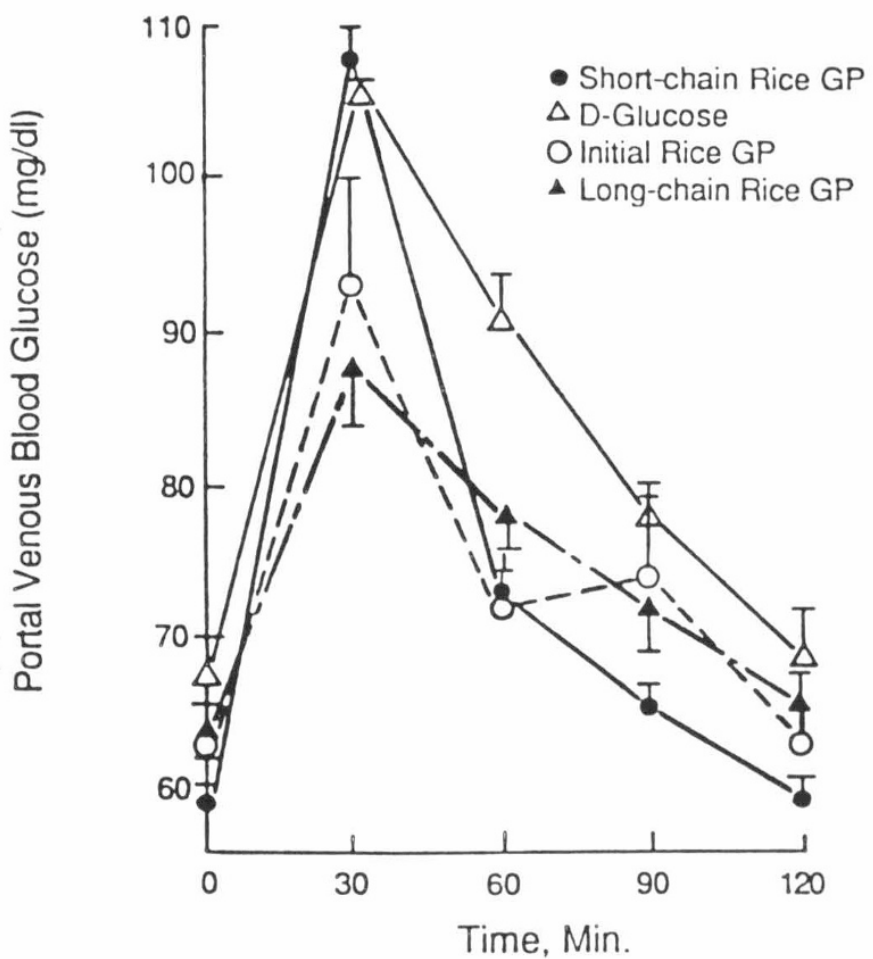

Fig. 2. Portal venous blood glucose response curves for short-chain rice GP $(\bullet)$, D-glucose $(\triangle)$, initial rice GP $(\bigcirc)$, and long-chain rice GP (A). All four carbohydrates produced maximal peaks at $30 \mathrm{~min}$. The maximal increase in portal blood glucose is significantly greater for shortchain rice GP infusion in comparison to the initial rice GP, long-chain rice GP. and D-glucose. Maximal increase in portal blood glucose after the initial rice GP infusion is not significantly different from that of Dglucose infusion. Data are expressed as mean values \pm SEM. of the glucoamylase using G5 to G8 units was found to be linear and constant for the entire $90 \mathrm{~min}$, whereas when using the glycogen as a substrate, there was linearity for $30 \mathrm{~min}$ and then an increase. Furthermore, the glucose production using G5 to G8 units compared to glycogen was higher during the 30 and 60 min of the reaction (Fig. 3). This observation is consistent with the finding that the reaction rate of glucoamylase increases with decreasing length of the substrate, glycogen.

Glycerol inhibition studies. Glycerol at 1.2 M concentration caused reductions in glucoamylase activities, using GP G5 to G8 units as substrate, to $45 \%$ of its activity under glycerol-free conditions, whereas using glycogen as the substrate resulted in a reduction to $40 \%$ of glucoamylase activity under the identical assay system (Fig. 3).

\section{DISCUSSION}

The main finding of our study indicates that short-chain GP of rice composed of $\mathrm{G} 2$ to $\mathrm{G} 9$ glucose units are hydrolyzed and absorbed in the small intestine faster than isocaloric D-glucose. Our results are similar to those in the study by Jones et al. (14) that was performed on humans but with corn GP. In their study, the intestinal absorption and mucosal hydrolysis of a partial and a complete $\alpha$-amylase hydrolysate of corn starch, simulating the normal intermediary and end products of luminal starch digestion, was undertaken using an in vivo steady state jejunal perfusion technique (excluding pancreatic $\alpha$-amylase) in normal human subjects. Whereas Jones et al. (14) used a $25-\mathrm{cm}$ jejunal segment, we used the whole small intestine, including pancreatic $\alpha$-amylase, simulating a real life situation for digestion and absorption processes of GP. In the study of Jones et al., hydrolysis of the polymer fraction containing more than 10 glucose units was significantly slower than the lower mol wt fraction, and it was postulated that oligosaccharides in the more rapidly hydrolyzed lower mol wt fraction were exerting a kinetic advantage on glucose absorption. Whereas Jones et al. (14) used luminal disappearance of the corn GP as evidence of GP hydrolysis and absorption, we measured the effect of GP hydrolysis and absorption as the portal venous blood glucose response. There is evidence that, in rats (15), most of the intraluminally absorbed glucose is absorbed intact into the portal vein and not metabolized to lactate. During peak absorption time, glucose concentration in the portal vein is higher than that in the aorta, suggesting a minimal role of insulin and other regulatory hormones in glucose homeostasis in portal venous blood (15).

The finding of lower total 120 -min glycemic response for the initial GP of rice in comparison to D-glucose and no significant difference of glycemic response values at 0 to $30 \mathrm{~min}$ is important. The explanation might be related to efficient hydrolysis of GP in the G2-G9 fraction of the initial rice polymer and the slower hydrolysis of the $>\mathrm{G} 9$ fraction that might be the limiting factor for efficient glycemic response. That is why we separated the initial rice GP to short-chain GP (G2-G9) and long-chain GP $(>\mathrm{G} 9)$ of rice. We found that the portal glycemic response was significantly higher for short-chain GP of rice in comparison to long-chain GP of rice both in the 0 to $30 \mathrm{~min}$ and in the total $120 \mathrm{~min}$.

The faster short-chain GP absorption in comparison to Dglucose during peak absorption time could be explained by the combined effects of the property of GP, its low osmolality in comparison to D-glucose, and the preferential substrate use by intestinal glucoamylase. GP, due to incorporation of water during hydrolysis, will yield more moles of hexose than the Dglucose solution of the same weight concentration. The osmolalities of our test GP solutions were much less than the D-glucose solution. Daum et al. (16) found a larger increment of intraduodenal water content, indicating flux of water, after $10 \%$ free Dglucose infusion compared with $10 \%$ GP infusion. Even at 30 min, $10 \%$ GP solution produced a lower luminal osmolality than that of $10 \%$ free glucose. This might be part of the explanation 
Table 1. Area under total portal blood glucose response curve (AUC) above fasting level after intraduodenal infusion of short-chain rice GP, long-chain rice GP, initial rice GP, and D-glucose*

\begin{tabular}{lcccc}
\multicolumn{1}{c}{ Time $(\mathrm{min})$} & \multicolumn{4}{c}{ Rise in portal venous blood glucose AUC $\left(\mathrm{cm}^{2}\right)$} \\
\cline { 2 - 5 } & $\begin{array}{c}\text { Short-chain GP } \\
(n=5)\end{array}$ & $\begin{array}{c}\text { Long-chain GP } \\
(n=5)\end{array}$ & $\begin{array}{c}\text { Initial rice GP } \\
(n=7)\end{array}$ & $\begin{array}{c}\text { D-glucose } \\
(n=6)\end{array}$ \\
\hline Total 120 & $2.5 \pm 0.1 \dagger$ & $1.2 \pm 0.2$ & $1.6 \pm 0.2$ & $2.0 \pm 0.1$ \\
First 1/2 h rise (\% of total) & $6.9 \pm 0.4$ & $4.6 \pm 0.6$ & $5.4 \pm 0.6 \ddagger$ & $7.5 \pm 0.5$ \\
First h rise (\% of total) & $35.9 \pm 1.3 \$$ & $26.0 \pm 2.8$ & $30.3 \pm 1.9$ & $27.5 \pm 1.1$ \\
\end{tabular}

* Values are presented as mean \pm SEM. The portal venous glycemic response at 0 to $30 \mathrm{~min}$ is significantly greater for short-chain rice GP infusion than for D-glucose. The total $120 \mathrm{~min}$ glycemic response is significantly lower for the initial rice GP infusion than for D-glucose infusion. The kinetics of absorption of tested GP, defined as the percentage of the total rise of the area under the portal blood glucose curve over time, reveals significantly higher values both at 1st $30 \mathrm{~min}$ and $1 \mathrm{st} 60 \mathrm{~min}$ for short-chain rice GP infusion in comparison to D-glucose infusion.

$\dagger p<0.01$.
$\ddagger p<0.02$.
$\$ p<0.001$.

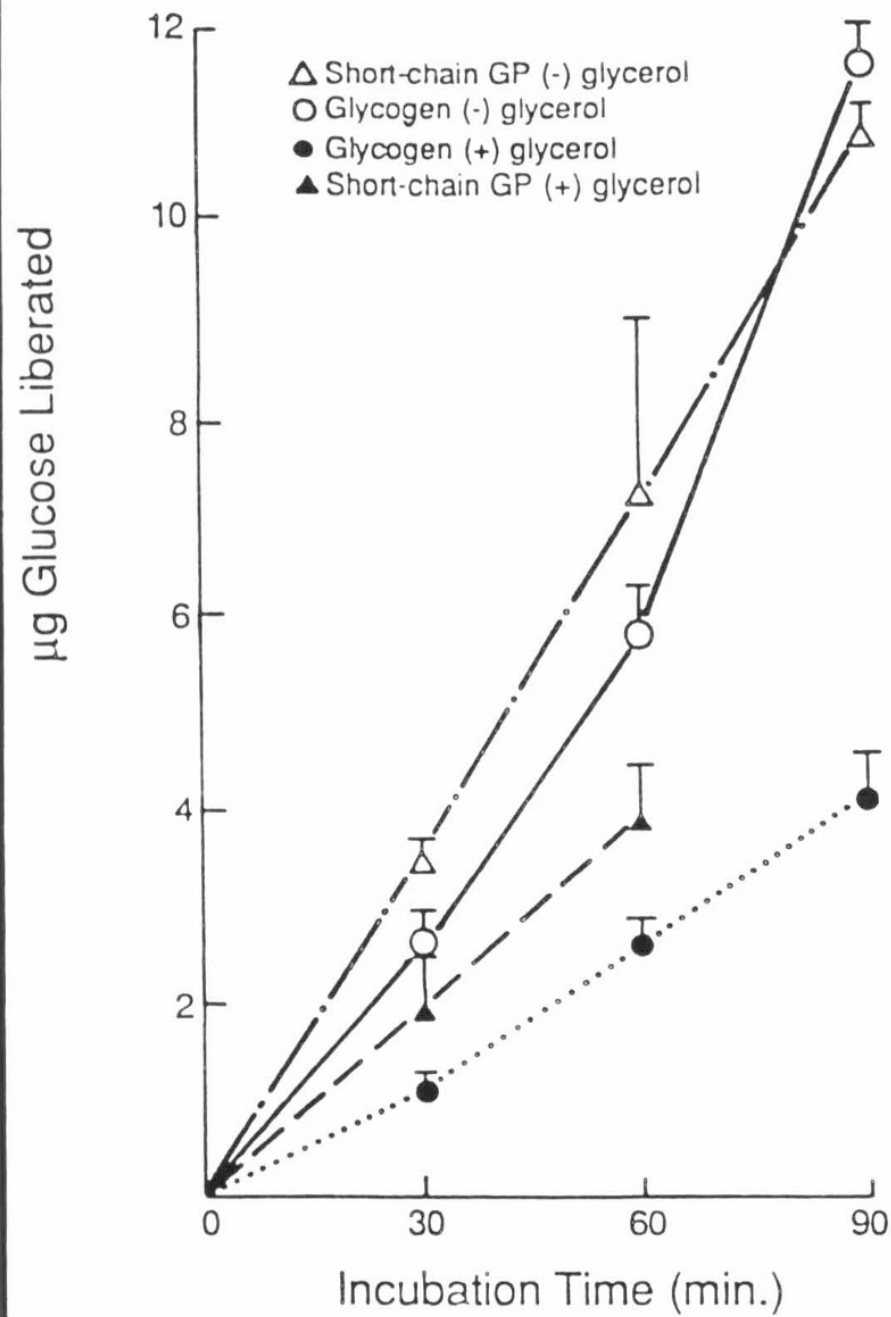

Fig. 3. Comparison of time course of glucoamylase assay using shortchain GP (G5-G8) and glycogen as substrates. The assay was carried out in absence $[(\triangle)$ short-chain GP, $(\bigcirc)$, glycogen] and presence $[(\mathbf{\Delta})$ shortchain GP. (-) glycogen] of glycerol. Five $\mu \mathrm{L}$ of mucosal homogenates were used for incubation. Assays were set up as described in Methods. Glycerol was added to a final concentration of $1.2 \mathrm{M}$ in the appropriate assay mixture. For each time point, two assay mixtures were prepared. Assay mixtures were incubated for the designated time. Activities were measured as $\mu \mathrm{g}$ of glucose liberated per assay mixture after the designated time of incubation. Values represent the mean $\pm 1 \mathrm{SD}$ for slower D-glucose absorption in comparison to GP absorption (17). We designed our study to compare the portal venous blood glucose response of the three GP solutions of different chainlengths and similar osmolalities with D-glucose infusion.

The finding of significantly higher portal venous blood glucose during peak absorption time with short-chain GP in comparison to the initial rice GP or the long-chain rice GP indicates that osmolality alone could not account for the observed difference. This might be an advantage of short-chain GP, when planning infant feeding designs where increased caloric density and lowered osmolality is desirable. One example of the practical relevance of faster short-chain GP absorption over D-glucose would be its use in short bowel syndrome and in patients with rapid intestinal transit time.

In contrast to the human, glucose absorption in the rat small intestine occurs by two independent routes. One is an active electrogenic component that is saturable (luminal glucose concentration $\sim 64 \mathrm{mM}$ ) and phlorizin sensitive. The other is a diffusional pathway that is nonelectrogenic, nonsaturable, and phlorizin insensitive. At high luminal glucose concentrations, diffusional component accounts for $50 \%$ of glucose absorption (18). The osmolality of our test glucose solution was 561 mos$\mathrm{mol} / \mathrm{L}$, which definitely superseded the active electrogenic saturable component. The portal venous blood glucose level might be the combination of both components. The finding of significantly higher glycemic response with short-chain GP at peak absorption time infers that hydrolysis of the short-chain GP might have yielded more glucose at the glucose transport sites than D-glucose. This is in agreement with the finding of Daum et al. (16) that GP produces a lower immediate intraduodenal glucose content (conversely higher mucosal glucose content) than a D-glucose solution of the same concentration by weight.

Due to difficulties correlating the in vivo perfusion results and in vitro enzyme assay in human subjects, our study was designed to correlate the activities of glucoamylase and glucose production in vitro by using short-chain GP (G5-G8) and glycogen as substrates. Glucoamylase is the key enzyme for brush-border hydrolysis of short-chain GP (G5-G9); the enzyme sp act decreases with either increasing or decreasing chain-length of the GP (19). We carried out and modified the glucoamylase assay by using the optimal substrate G5-G8, as well as glycogen (prototype of long-chain rice GP), as used in the past $(9,11)$.

The finding of a linear time-course for glucoamylase activity in the mucosal homogenates over the entire 90 -min period with short-chain GP (G5-G8) as substrate in comparison to glycogen indicates preferential use of short-chain GP (G5-G8) by glucoamylase. This is also an advantage of our modification of the glucoamylase assay. This is in agreement with the finding of Kelly and Alpers (19) for human intestinal glucoamylase, where they found linear GP linkages (alpha 1-4) were hydrolyzed more 
rapidly than polymers containing (alpha 1-6) cross linkages. They also found a similar $\mathrm{Km}$ for all polymers from G5 to G9 and the increase of $\mathrm{Km}$ value with either an increase of polymer length $(>\mathrm{G} 9)$ or a decrease of polymer length $(<\mathrm{G} 5)$.

The current cost of D-glucose is to some extent prohibitive in lesser developed countries. It is feasible that the use of less expensive, short-chain GP will be more easily afforded by lesser developed countries,

In conclusion, short-chain rice GP with a low osmolality, compared with isocaloric D-glucose, may be an advantageous energy source for children with chronic diarrhea in developing countries.

Acknowledgments. The authors thank P. C. Lee, Ph.D. and Bridget Krantz, B.Sc. for their suggestions and technical assistance. We also thank Pauline Kosmoski for secretarial assistance.

\section{REFERENCES}

1. Auricchio S, Pietra DD. Vegnente A 1967 Studies on intestinal digestion of starch in man. II. Intestinal hydrolysis in infants and children. Pediatrics 39:853-862

2. Gray GM 1970 Carbohydrate digestion and absorption. Gastroenterology 58:96-107

3. Lebenthal E 1978 Pancreatic function and disease in infancy and childhood. Adv Pediatr 25:223-261

4. Lebenthal E, Lee PC 1980 Development of functional response in human exocrine pancreas. Pediatrics 66:556-560

5. Lifschitz F 1977 The enteric flora in childhood disease-diarrhea. Am J Clin Nutr 30:1811-1818

6. Rossi TM, Lebenthal E. Nord KS, Fazili RR 1980 Extent and duration of small intestinal mucosal injury in intractable diarrhea of infancy. Pediatrics $66: 730-735$
7. Shwachman H, Lloyd-Still JD. Khaw KT, Antonowicz I 1973 Protracted diarrhoea of infancy treated by intravenous alimentation. II. Studies of small intestinal biopsy results. Am J Dis Child 125:365-368

8. Webb JD, Poley JR. Bhatia M. Stevenson DE 1978 Intestinal disaccharidase activities in relation to age, race and mucosal damage. Gastroenterology 75:847-855

9. Lebenthal E, Lee PC 1980 Glucoamylase and disaccharidase activity in normal subjects and in patients with mucosal injury of the small intestine. J Pediatrics 97:389-393

10. John M. Trenel G, Dellweg H 1969 Quantitative chromatography of homologous glucose oligomers and other saccharides using polyacrylamide gel. J Chromatogr 42:476-484

11. Eggermont E 1969 The hydrolysis of the naturally occurring alpha glucosides by the human intestinal mucosa. Eur J Biochem 9:483-487

12. Townley RR, Khaw KT, Shwachman H 1965 Quantitative assay of disaccharidase activities of small intestinal mucosal biopsy specimens in infancy and childhood. Pediatrics 36:911-921

13. Lowry OH, Rosebrough NJ, Farr AL, Randall RJ 1951 Protein measurement with the folin phenol reagent. J Biol Chem 193:265-277

14. Jones BJM, Brown BE, Loran JS, Edgerton D. Kennedy JF. Stead JA, Silk DBA 1983 Glucose absorption from starch hydrolysates in the human jejunum. Gut 24:1152-1160

15. Rich-Densen C. Kimura RE 1988 Evidence in-vivo that most of the intraluminally absorbed glucose is absorbed intact into the portal vein and not metabolized to lactate. Biochem J 254:931-934

16. Daum F, Cohen MI, McNamara H. Finberg L 1978 Intestinal osmolality and carbohydrate absorption in rats treated with polymerized glucose. Pediatr Res 12:24-26

17. Fullerton PM, Parsons DS 1956 The absorption of sugars and water from rat intestine in vivo. Q J Exp Physiol 41:387-397

18. Debnam ES, Levin RJ 1975 An experimental method of identifying and quantifying the active transfer electrogenic component from the diffusive component during sugar absorption measured in vivo. J Physiol (Lond) 246:181-196

19. Kelly JJ, Alpers DH 1973 Properties of human intestinal glucoamylase. Biochim Biophys Acta 315:113-129 